\title{
Bell's Palsy
}

National Institute of Neurological Disorders and Stroke (NINDS)

\section{Source}

National Institute of Neurological Disorders and Stroke (NINDS). Bell's Palsy Information

Page.

Bell's palsy is a form of temporary facial paralysis resulting from damage or trauma to the 7th cranial nerve, one of the facial nerves. It is the most common cause of facial paralysis. Generally, Bell's palsy affects only one side of the face, but it can affect both sides. Symptoms vary among individuals and include sudden weakness on one side of the face, drooping eyelid or corner of the mouth, drooling, inability to close the eye or mouth, altered taste, and excessive tearing in the eye. Symptoms appear suddenly and reach their peak within 72 hours. Bell's palsy can range in severity from mild weakness to total paralysis, and can cause significant facial distortion. The exact cause of Bell's palsy isn't known, but many scientists believe that reactivation of a dormant viral infection can cause the facial nerve to swell and become inflamed. Several other conditions can cause facial paralysis that might be diagnosed as Bell's palsy. The disorder can affect anyone at any age. Risk factors include pregnancy, preeclampsia, obesity, hypertention, diabetes, and upper respiratory ailments. 